This item was submitted to Loughborough's Research Repository by the author.

Items in Figshare are protected by copyright, with all rights reserved, unless otherwise indicated.

\title{
Sub-terahertz amplification in a semiconductor superlattice with moving charge domains
}

PLEASE CITE THE PUBLISHED VERSION

http://dx.doi.org/10.1063/1.4906531

\section{PUBLISHER}

(c) American Institute of Physics

\section{VERSION}

VoR (Version of Record)

\section{PUBLISHER STATEMENT}

This work is made available according to the conditions of the Creative Commons Attribution-NonCommercialNoDerivatives 4.0 International (CC BY-NC-ND 4.0) licence. Full details of this licence are available at: https://creativecommons.org/licenses/by-nc-nd/4.0/

\section{LICENCE}

CC BY-NC-ND 4.0

\section{REPOSITORY RECORD}

Makarov, V.V., Alexander E. Hramov, Alexey A. Koronovskii, Kirill N. Alekseev, V.A. Maximenko, M.T. Greenaway, T.M. Fromhold, Olga I. Moskalenko, and Alexander G. Balanov. 2019. "Sub-terahertz Amplification in a Semiconductor Superlattice with Moving Charge Domains". figshare. https://hdl.handle.net/2134/17298. 


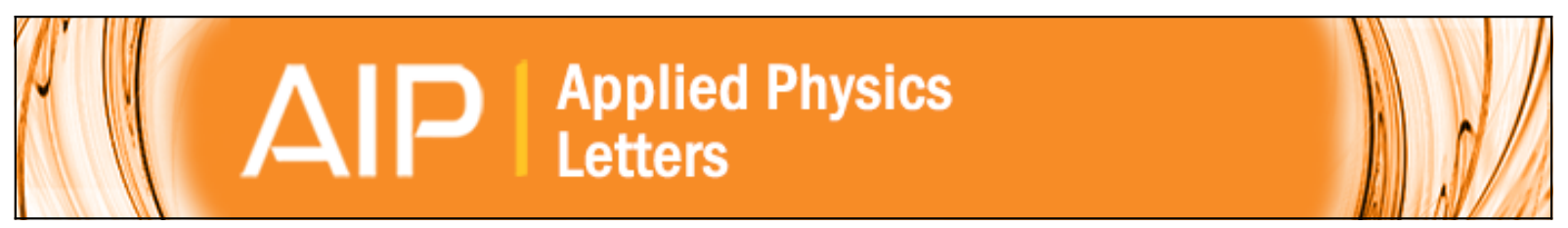

\section{Sub-terahertz amplification in a semiconductor superlattice with moving charge domains}

V. V. Makarov, A. E. Hramov, A. A. Koronovskii, K. N. Alekseev, V. A. Maximenko, M. T. Greenaway, T. M. Fromhold, O. I. Moskalenko, and A. G. Balanov

Citation: Applied Physics Letters 106, 043503 (2015); doi: 10.1063/1.4906531

View online: http://dx.doi.org/10.1063/1.4906531

View Table of Contents: http://scitation.aip.org/content/aip/journal/apl/106/4?ver=pdfcov

Published by the AIP Publishing

\section{Articles you may be interested in}

Terahertz radiation induced chaotic electron transport in semiconductor superlattices with a tilted magnetic field

Chaos 24, 033109 (2014); 10.1063/1.4890240

Terahertz parametric gain in semiconductor superlattices in the absence of electric domains

Appl. Phys. Lett. 89, 132105 (2006); 10.1063/1.2357579

Frequency multiplication of microwave radiation by propagating space-charge domains in a semiconductor superlattice

Appl. Phys. Lett. 82, 2826 (2003); 10.1063/1.1569999

Current suppression and harmonic generation by intense terahertz fields in semiconductor superlattices

J. Appl. Phys. 82, 718 (1997); 10.1063/1.365604

Terahertz-field-induced recovery of resonant electron transparency of semiconductor superlattices in electric field J. Appl. Phys. 81, 1771 (1997); 10.1063/1.364032

You don't

still use this

cell phone

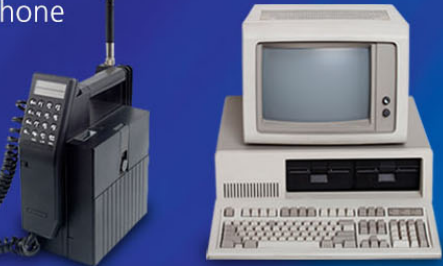

Why are you still using an AFM designed in the 80 's?
It is time to upgrade your AFM

Minimum $\$ 20,000$ trade-in discount for purchases before August 31st

Asylum Research is today's technology leader in AFM 


\title{
Sub-terahertz amplification in a semiconductor superlattice with moving charge domains
}

\author{
V. V. Makarov, ${ }^{1,2}$ A. E. Hramov, ${ }^{1,2}$ A. A. Koronovskii, ${ }^{1,2}$ K. N. Alekseev, ${ }^{3}$ V. A. Maximenko, ${ }^{1,2}$ \\ M. T. Greenaway, ${ }^{4}$ T. M. Fromhold, ${ }^{4}$ O. I. Moskalenko, ${ }^{1,2}$ and A. G. Balanov ${ }^{3}$ \\ ${ }^{1}$ Saratov State Technical University, Politechnicheskaja 77, Saratov 410054, Russia \\ ${ }^{2}$ Faculty of Nonlinear Processes, Saratov State University, Astrakhanskaya 83, Saratov 410012, Russia \\ ${ }^{3}$ Department of Physics, Loughborough University, Loughborough LE11 3TU, United Kingdom \\ ${ }^{4}$ School of Physics and Astronomy, University of Nottingham, Nottingham NG7 2RD, United Kingdom
}

(Received 9 December 2014; accepted 13 January 2015; published online 29 January 2015)

\begin{abstract}
We theoretically study the high-frequency response of charge domains traveling through a strongly coupled semiconductor superlattice with an applied harmonic electromagnetic signal. Our calculations show that the superlattice alone can amplify signals with a frequency close to the domain transient frequency. Moreover, we show that if the superlattice is connected to a resonator, amplification becomes possible for much higher frequencies of the external signal (several hundred $\mathrm{GHz}$ ). These promising results open the way to using semiconductor superlattices as efficient subTHz amplifiers. (C) 2015 AIP Publishing LLC. [http://dx.doi.org/10.1063/1.4906531]
\end{abstract}

Devices that operate in the sub-terahertz and terahertz range $(0.1-10 \mathrm{THz})$ of the electromagnetic spectrum are crucially important for many prominent applications in key areas of science and technology such as astrophysics, broadband communications, medicine, and security. ${ }^{1-3}$ However, present $\mathrm{THz}$ technologies are still severely limited (a phenomenon known as the THz gap). ${ }^{4}$ One of the principle reasons for this is the lack of reliable compact solid state sources and amplifiers able to work in this frequency range. ${ }^{5,6}$ Although considerable progress has been recently achieved in the buildout of sub-THz/THz sources, ${ }^{7-10}$ the development of amplifiers of sub- $\mathrm{THz} / \mathrm{THz}$ signals still remains a significant challenge. 5,11

In this paper, we explore the feasibility of using semiconductor superlattices (SLs) ${ }^{12,13}$ for the amplification of electromagnetic waves in the sub- $\mathrm{THz}$ range when moving charge domains are present in the device. ${ }^{14-19}$ SLs comprise multiple alternating layers of different semiconductor materials, ${ }^{12,13}$ which form a periodic modulation of the conduction band. This creates a tunable, quantum mechanical environment that is suitable for the realization of Bloch gain, ${ }^{7,20-23}$ which can be utilized both for the generation and amplification of sub- $\mathrm{THz}$ and $\mathrm{THz}$ signals. However, the same quantum mechanisms that give rise to Bloch gain result in electric instability, which leads to the formation of moving high-field charge domains. ${ }^{24}$ This induces electric field inhomogeneity that destroys Bloch gain and makes amplification impossible. ${ }^{25}$ Recently, research has been focused on the development of approaches that suppress the emergence of charge domains in SLs. ${ }^{25-28}$ Here, we propose to employ moving charge domains as an active medium for the amplification of high-frequency signals in SLs. This work is motivated by earlier research on Gunn diodes, ${ }^{29,30}$ where amplification was shown to be feasible for signals with frequencies both below ${ }^{29}$ and above ${ }^{30}$ the transient frequency of the Gunn domains. Here, we examine if charge domains in SLs are able to provide gain for high-frequency signals and whether a resonator can improve the characteristics of the SL amplifier.
The system under study is shown schematically in Fig. 1. The device consists of a SL mounted into a coaxial resonator and ferrite circulator ${ }^{31}$ that separates the input and output signals. A strongly coupled SL, where electron transport occurs within a single miniband, is used as the active element. To describe the charge dynamics in the SL, we use a set of 1D time-dependent current continuity and Poisson equations, which self-consistently couple the volume electron density, $n$, the electric field along the SL, $F$, and the local current density, $J$ (Ref. 24)

$$
\begin{gathered}
\frac{\partial n(x, t)}{\partial t}=-\frac{1}{e} \frac{\partial J(x, t)}{\partial x} \\
\frac{\partial F(x, t)}{\partial x}=\frac{e}{\varepsilon_{0} \varepsilon_{r}}\left[n(x, t)-n_{D}\right] .
\end{gathered}
$$

Here, $x$ is the spatial coordinate perpendicular to the SL layers, $t$ is time, $n_{D}$ is the n-type doping density in the SL layers, $e>0$ is the electron charge magnitude, and $\varepsilon_{0}$ and $\varepsilon_{r}$
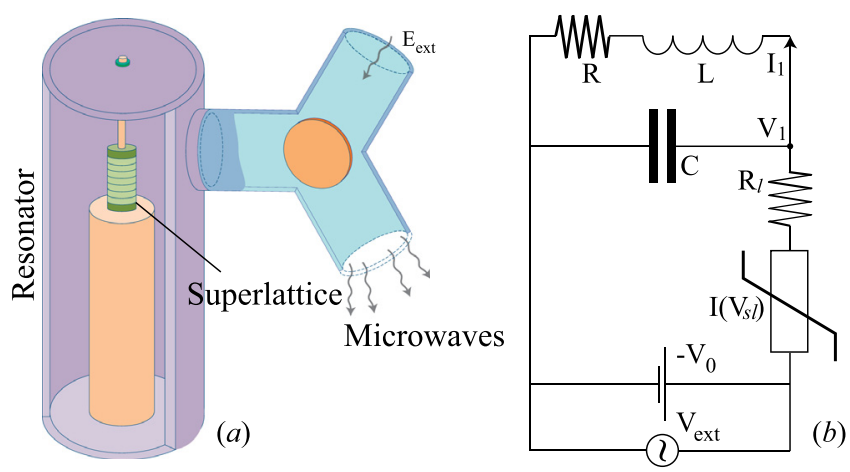

FIG. 1. (a) Schematic diagram of a semiconductor superlattice coupled to an external EM resonator and driven by an external electromagnetic field $E_{\text {ext }}$. (b) Equivalent circuit for a SL interacting with an external single-mode resonator driven by the external signal. Here $C, L$, and $R$ are the equivalent capacitance, inductance, and resistance of the resonator, $V_{s l}$ is the voltage dropped across the SL, $I\left(V_{s l}\right)$ is the current through the SL, $V_{0}$ is the DC supply voltage, and $V_{\text {ext }}$ is AC voltage resulting from the applied electromagnetic radiation. 
are the absolute and relative permittivities, respectively. The current density is calculated using $J=e n v_{d}(F)$, where the drift velocity, $v_{d}$, of miniband electrons for a miniband width, $\Delta$, and temperature, $T$, can be estimated analytically ${ }^{32}$

$$
v_{d}(F)=\frac{\Delta d}{2 \hbar} \frac{I_{1}\left(\Delta / 2 k_{B} T\right)}{I_{0}\left(\Delta / 2 k_{B} T\right)} \frac{e F \tau d / \hbar}{1+(\tau e F d / \hbar)^{2}} .
$$

Equation (3) is obtained by averaging of the Esaki-Tsu formula for drift velocity ${ }^{12}$ with respect to the MaxwellBoltzmann distribution of the initial electron momentums at the given temperature $T .^{33}$ Here, $I_{0,1}($.$) are the modified$ Bessel functions of the first kind, $k_{B}$ and $\hbar$ are the Boltzmann and the Planck constants, and $d$ is the spatial period of the SL.

Electron scattering in the SLs is characterized by the effective scattering time, $\tau$, which takes into account both elastic and inelastic events. ${ }^{24} \mathrm{We}$ assume Ohmic contacts, which determine the boundary conditions $J(0, t)=\sigma F(0, t)$, where $\sigma$ is the contact conductivity and the voltage $V_{s l}$ applied across the SL defines a global constraint to the system

$$
V_{s l}=U+\int_{0}^{L} F(x) d x,
$$

where $L$ is the length of the transport region in the SL. The voltage drop across the contacts, $U$, includes the effect of charge accumulation and depletion in the emitter and collector regions and a contact resistance, as shown in Refs. 34 and 35 . The total current through the SL with a cross-sectional area $A$ is then given by ${ }^{24}$

$$
I(t)=\frac{A}{L} \int_{0}^{L} J(x, t) d x .
$$

In our calculations, we take parameters corresponding to SLs used in recent experiments: ${ }^{36-38} d=8.3 \mathrm{~nm}, L=115.2 \mathrm{~nm}, A$ $=5 \times 10^{-10} \mathrm{~m}^{2}, \Delta=19.1 \mathrm{meV}, n_{D}=3 \times 10^{22} \mathrm{~m}^{-3}, \tau=250 \mathrm{fs}$, $\varepsilon_{r}=12.5, \sigma=3788 \mathrm{Sm}^{-1}$, and $T=4.2 \mathrm{~K}$. We solved the model equations (1)-(5) numerically using the discretization scheme discussed in Refs. 35 and 36.

First, we investigate gain in the SL alone, i.e., without a resonator. The current-voltage curve of the device is shown in the inset of Fig. 2. The descending part of this curve (for $V_{s l}>V_{t h} \approx 360 \mathrm{mV}$ ), or negative differential conductance (NDC), is responsible for the emergence of moving charge domains. These domains generate current oscillations with a rich spectral content. For example, in Fig. 2, the amplitude spectrum of the current oscillations is presented for $V_{s l}=510 \mathrm{mV}$. Remarkably, although the fundamental frequency of the current oscillations $f_{0} \approx 17.3 \mathrm{GHz}$, there is also significant power associated with harmonic frequencies in the range $0.2-1 \mathrm{THz}$.

The action of an external monochromatic electromagnetic field can be considered as an additional $\mathrm{AC}$ voltage applied to the SL, so that $V_{s l}=V_{0}+V_{\text {ext }} \cos \left(2 \pi f_{e x t} t\right)$, where $V_{0}$ represents a DC bias, while $V_{\text {ext }}$ and $f_{\text {ext }}$ are the amplitude and

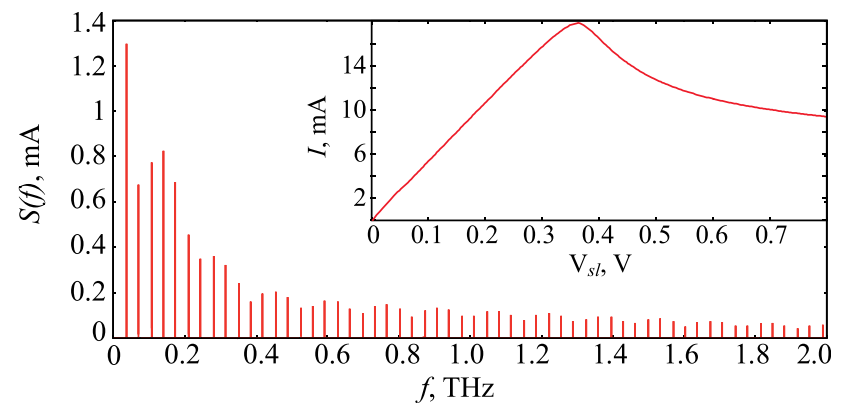

FIG. 2. Amplitude spectrum of current oscillations in a single superlattice (without resonator) for $V_{s l}=510 \mathrm{mV}$. Inset: Current-voltage characteristics $I\left(V_{s l}\right)$.

frequency of the external signal. In this case, the absorption of the AC field ${ }^{39}$ can be introduced within small signal approximation as $\alpha\left(f_{\text {ext }}\right)=\Re\left[\sigma\left(f_{\text {ext }}\right)\right] /\left(n_{r} \varepsilon_{0} c_{0}\right)$, where $\Re\left[\sigma\left(f_{\text {ext }}\right)\right]$ $\approx 2\left(\left\langle I(t) \cos \left(2 \pi f_{\text {ext }} t\right)\right\rangle_{t} / A\right) /\left(V_{\text {ext }} / L\right)$ is real part of the highfrequency conductivity, ${ }^{26}\langle$.$\rangle represents the time average over$ infinity, $n_{r}$ is the refractive index of the SL material, and $c_{0}$ is the speed of light in vacuum.

Figure 3(a) shows the dependence of $\alpha$ upon $f_{\text {ext }}$ for $V_{0}=510 \mathrm{mV}$ and $V_{e x t}=60 \mathrm{mV}$. The plots reveal amplification of the signal $(\alpha<0)$ when $f_{\text {ext }}<9 \mathrm{GHz}$. In this $f_{\text {ext }}$ range, the increase of $V_{s l}$ with increase in time enhances the field inside the moving charge domain, and thus localizes the charge carriers. This means that the current decreases until the high field in the domain reaches a maximum value. Hence, an additional dynamic (high-frequency) NDC is generated in the SL, which promotes the amplification of highfrequency signals. However, this process is only possible when the applied $\mathrm{AC}$ voltage has a frequency low enough to allow the domain enough time to respond to the perturbation, and therefore, we find $\alpha>0$ for $f_{\text {ext }}>9 \mathrm{GHz}$.

We also reveal peaks in the absorption at frequency intervals corresponding to synchronization within the system, i.e., when the frequency of the domain transition is
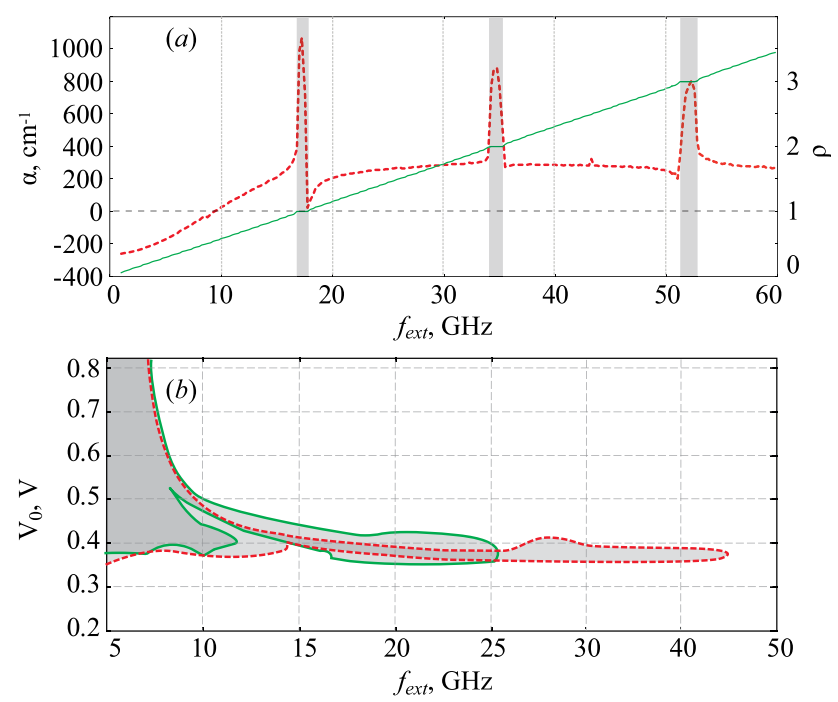

FIG. 3. (a) $\alpha\left(f_{\text {ext }}\right)$ in a single SL (dashed line, red online) and $\rho\left(f_{\text {ext }}\right)$ (solid line, green online) for $V_{0}=510 \mathrm{mV}$ and $V_{\text {ext }}=20 \mathrm{mV}$. Synchronization regions are indicated by grey stripes. (b) Areas of amplification, $\alpha<0$, in the parameter plane $\left(f_{e x t}, V_{0}\right)$ for $V_{e x t}=20 \mathrm{mV}$ (dashed contour, red online) and $V_{e x t}=60 \mathrm{mV}$ (solid contour, green online). 
locked by the frequency of the external signal. To illustrate this, we calculate the rotation number ${ }^{40} \rho=f_{\text {ext }} / f_{0}$, which is represented by the solid curve in Fig. 3(a). The $\rho\left(f_{\text {ext }}\right)$ curve has characteristic plateaus with values $\rho=1,2,3, \ldots$, each time $\alpha\left(f_{e x t}\right)$ exhibits a prominent peak: see regions highlighted by grey vertical stripes.

Figure 3(b) shows the regions of amplification in the parameter plane $\left(f_{\text {ext }}, V_{0}\right)$ for $V_{\text {ext }}=20 \mathrm{mV}$ (dashed contour, red online) and $V_{e x t}=60 \mathrm{mV}$ (solid contour, green online). In both cases, amplification is possible only in the presence of moving charge domains, i.e., for $V_{0}>V_{t h}$, where $V_{t h} \approx 360 \mathrm{mV}$ is the threshold voltage corresponding to onset of current oscillations. For large $V_{0}$, amplification is completely suppressed, since-for high DC bias-the domains no longer respond to the AC component of the applied voltage, and thus, high-frequency NDC is destroyed. Notably, in both cases, amplification is realised for frequencies that are close to the first harmonic in the current oscillations spectrum (see Fig. 2). However, the presence of prominent high-frequency spectral components suggests that tuning the resonator to them may make the space-charge domains more sensitive to high-frequency signals.

To investigate this hypothesis, we consider the SL interacting with a single-mode external resonator, which is characterised by a resonant frequency, $f_{Q}$, and quality factor $Q$. The action of such a resonator upon the SL can be represented by an equivalent RLC-circuit shown in Fig. 1(b). In this case, the model equations (1)-(5) should be coupled to the non-stationary Kirchhoff equations

$$
\begin{gathered}
C \frac{d V_{1}}{d t}=I\left(V_{s l}\right)-I_{1}, \\
L \frac{d I_{1}}{d t}=R I_{1}+R_{l} I\left(V_{s l}\right)-V_{1},
\end{gathered}
$$

where $L, C$, and $R$ are the inductance, capacitance, and resistance of the resonant circuit, and $R_{l}$ is the load resistance. The variables $V_{1}$ and $I_{1}$ are the voltage across the capacitor and the current through the inductance, and $I\left(V_{s l}\right)$ is the current generated by the SL, where $V_{s l}=V_{1}+V_{0}+V_{e x t} \cos \left(2 \pi f_{e x t}\right)$. Plots of $\alpha\left(f_{\text {ext }}\right)$ and $\rho\left(f_{\text {ext }}\right)$ are shown in Fig. 4 calculated when $Q=750$ for various $f_{Q}$ corresponding to the first (a), second (b), and fourth (c) harmonics of $f_{0}$. Figure 4(a) reveals that tuning the resonator to the first harmonic improves amplification at the frequencies close to $f_{0}$, and also creates the possibility for amplification near higher harmonics of the current oscillations. For $f_{Q}$ close to the higher harmonics of $f_{0}$, we find that there is an increase in the the frequency of the amplified signal [see Figs. 4(b) and 4(c)]. Remarkably, the onset of synchronization significantly affects the absorption $\alpha$. We find that synchronization can lead either to increase of the absorption $(\alpha>0)$ or to amplification of the signal $(\alpha<0)$. Whether $\alpha>0$ or $\alpha<0$ depends on the phase difference between the external signal and the current oscillations generated in the SL. A full understanding of this phenomenon requires further study, which will be published elsewhere.

To gain deeper insight into the effect of $f_{Q}$ on $\alpha$, we superimpose the areas of the amplification $(\alpha<0)$ in the $\left(f_{Q}, f_{\text {ext }}\right)$ parameter plane on a color map, showing the

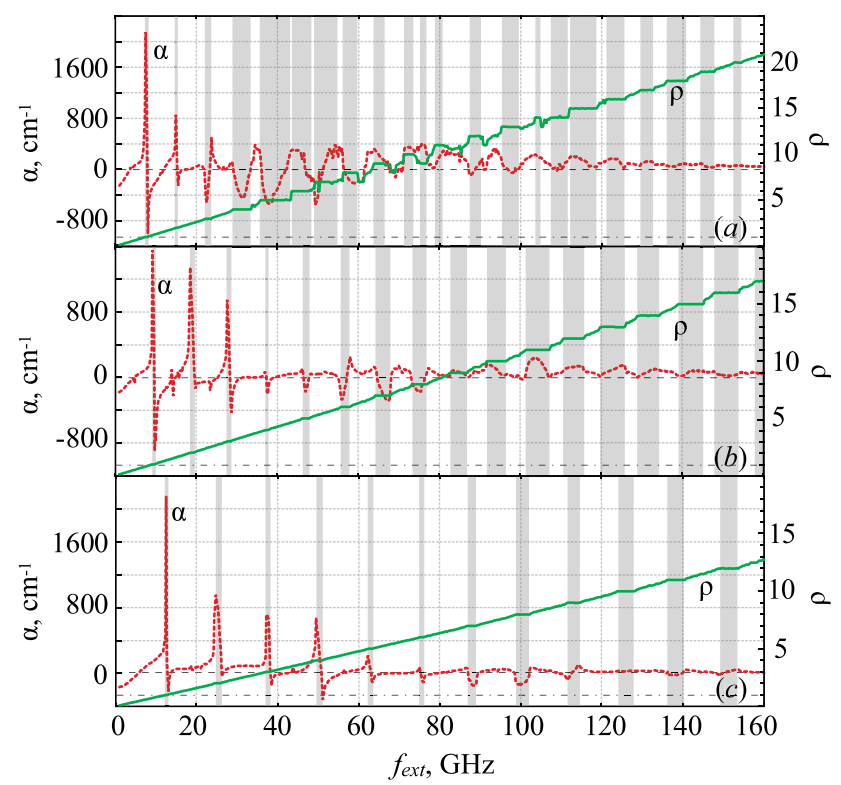

FIG. 4. $\alpha\left(f_{\text {ext }}\right)$ (dashed line, red online) and $\rho\left(f_{\text {ext }}\right)$ (solid line, green online) in a SL coupled to a resonator, when $V_{0}=510 \mathrm{mV}, V_{e x t}=20 \mathrm{mV}$, and $Q=750$. Synchronization regions are indicated by grey stripes. The frequency of the external resonator is (a) $f_{Q}=f_{0}$, (b) $f_{Q}=2 f_{0}$, and (c) $f_{Q}=4 f_{0}$, where $f_{0}=17.3 \mathrm{GHz}$ is the fundamental frequency of the current oscillations for the given $V_{0}$.

amplitude spectrum, $S(f)$, of the current oscillations (see Fig. 5) calculated for a range of $f_{Q}$. In this figure, yellow/ black corresponds to high/low power density. The regions of amplification are shown light grey. Figure 5 reveals that amplification is achieved when $f_{Q}$ is close to the harmonics in the current oscillation spectrum. Increasing $f_{Q}$ involves in the amplification the higher harmonics, thus enabling gain for signals whose frequency is much higher than the fundamental frequency of the current oscillations in the SL.

In conclusion, we have shown that propagating charge domains in SLs can be utilized as a medium for the amplification of sub-THz signals. We find that such a medium provides gain for signals with frequencies less than, or comparable to, the frequency of the current oscillations, $f_{0}$. Appropriate coupling of the SL to a resonator tuned to the harmonics of $f_{0}$ can significantly (several times) increase the gain provided by the moving charge domains. In addition,

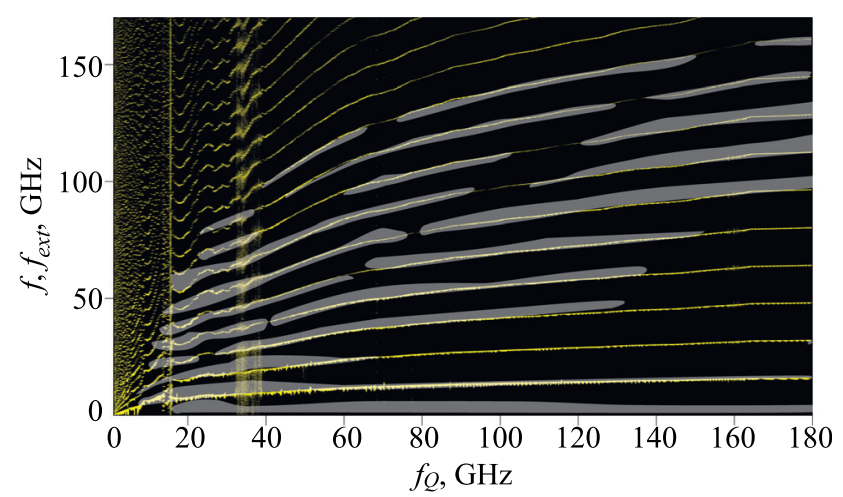

FIG. 5. Amplitude spectrum of the current oscillations, $S(f)$ as a function of $f_{Q}$ and the regions of $\alpha<0$ in $\left(f_{Q}, f_{e x t}\right)$ plane for $V_{0}=510 \mathrm{mV}$ and $V_{\text {ext }}=20 \mathrm{mV}$. Yellow denotes high power density, and black corresponds to low power density of the oscillations. Amplification regions are shown as light grey areas. 
the resonator can create conditions for the amplification of signals with frequencies close to higher harmonics of $f_{0}$. Our results provide a promising way to use semiconductor SLs as efficient sub-THz and $\mathrm{THz}$ amplifiers.

Russian Scientific Foundation supported study of the semiconductor superlattice coupled to resonator (Grant No. 14-12-00222). M.T.G. acknowledges financial support from the Leverhulme Trust (UK). We are thankful to Miron Kagan and Timo Hyart for useful discussions at the early stage of this work.

${ }^{1}$ S. Koenig, D. Lopez-Diaz, J. Antes, F. Boes, R. Henneberger, A. Leuther, A. Tessmann, R. Schmogrow, D. Hillerkuss, R. Palmer, T. Zwick, C. Koos, W. Freude, O. Ambacher, J. Leuthold, and I. Kallfass, Nat. Photonics 7, 977-981 (2013).

${ }^{2}$ M. Tonouchi, Nat. Photonics 1, 97-105 (2007).

${ }^{3}$ B. Ferguson and X.-C. Zhang, Nat. Mater. 1, 26-33 (2002).

${ }^{4}$ H.-T. Chen, W. J. Padilla, J. M. O. Zide, A. C. Gossard, A. J. Taylor, and R. D. Averitt, Nature 444, 597-600 (2006).

${ }^{5}$ S. R. Tripathi, Y. Taira, S. Hayashi, K. Nawata, K. Murate, H. Minamide, and K. Kawase, Opt. Lett. 39(6), 1649-1652 (2014); S. Sarkozy, J. Drewes, K. M. K. H. Leong, R. Lai, X. B. Mei, W. Yoshida, M. D. Lange, J. Lee, and W. R. Deal, Opt. Eng. 51, 091602 (2012).

${ }^{6}$ L. A. Samoska, IEEE Trans. Terahertz Sci. Technol. 1(1), 9-24 (2011).

${ }^{7}$ R. Terazzi, T. Gresch, M. Giovannini, N. Hoyler, N. Sekine, and J. Faist, Nat. Phys. 3, 329-333 (2007).

${ }^{8}$ P. Khosropanah, A. Baryshev, W. Zhang, W. Jellema, J. N. Hovenier, J. R. Gao, T. M. Klapwijk, D. G. Paveliev, B. S. Williams, S. Kumar, Q. Hu, J. L. Reno, B. Klein, and J. L. Hesler, Opt. Lett. 34(19), 2958-2960 (2009).

${ }^{9}$ H. Eisele, Electron. Lett. 46(6), 422-423 (2010).

${ }^{10}$ M. Feiginov, H. Kanaya, S. Suzuki, and M. Asada, Appl. Phys. Lett. 104, 243509 (2014).

${ }^{11}$ N. Jukam, S. S. Dhillon, D. Oustinov, J. Madeo, Ch. Manquest, S. Barbieri, C. Sirtori, S. P. Khanna, E. H. Linfield, A. G. Davies, and J. Tignon, Nat. Photonics 3, 715-719 (2009).

${ }^{12}$ L. Esaki and R. Tsu, IBM J. Res. Dev. 14, 61-65 (1970).

${ }^{13} \mathrm{R}$. Tsu, Superlattices to Nanoelectronics (Elsevier, 2005).

${ }^{14}$ A. A. Ignatov and Yu. A. Romanov, Physica Status Solidi B 73(1), 327-333 (1976).

${ }^{15}$ E. Schomburg, T. Blomeier, A. A. Ignatov, K. F. Renk, D. G. Paveliev, Yu. Koschurinov, V. Ustinov, A. Zhukov, S. Ivanov, and P. S. Kop'ev, Appl. Phys. Lett. 71, 401-403 (1997).

${ }^{16}$ M. W. Feise and D. S. Citrin, Appl. Phys. Lett. 75, 3536 (1999).
${ }^{17}$ V. L. Vaks, A. N. Panin, S. A. Basov, A. V. Illyuk, S. I. Pripolzin, D. G. Peveliev, and Yu. I. Koshurinov, Radiophys. Quantum Electron. 52(7), 511-517 (2009).

${ }^{18}$ H. Eisele, S. P. Khanna, and E. H. Linfield, Appl. Phys. Lett. 96, 072101 (2010).

${ }^{19}$ V. I. Sankin, A. V. Andrianov, A. O. Zakhar'in, and A. G. Petrov, JETP Lett. 94(5), 362-365 (2011).

${ }^{20}$ P. G. Savvidis, B. Kolasa, G. Lee, and S. J. Allen, Phys. Rev. Lett. 92(19), 196802 (2004).

${ }^{21}$ Y. Shimada, K. Hirakawa, M. Odnoblioudov, and K. A. Chao, Phys. Rev. Lett. 90, 046806 (2003).

${ }^{22}$ E. Schomburg, N. V. Demarina, and K. F. Renk, Phys. Rev. B 67, 155302 (2003).

${ }^{23}$ X. Lei, D. Wang, Z. Wu, and M. M. Dignam, Appl. Phys. Lett. 105, 062112 (2014).

${ }^{24}$ A. Wacker, Phys. Rep. 357, 1-111 (2002).

${ }^{25}$ H. Kroemer, eprint arXiv:cond-mat/0009311v1 [cond-mat.mes-hall].

${ }^{26}$ T. Hyart, K. N. Alekseev, and E. V. Thuneberg, Phys. Rev. B 77, 165330 (2008).

${ }^{27}$ T. Hyart, A. V. Shorokhov, and K. N. Alekseev, Phys. Rev. Lett. 98, 220404 (2007).

${ }^{28}$ T. Hyart, J. Mattas, and K. N. Alekseev, Phys. Rev. Lett. 103, 117401 (2009).

${ }^{29}$ H. W. Thim, IEEE. Trans. Electron Devices 14(9), 517-522 (1967).

${ }^{30}$ I. V. Altukhov, M. S. Kagan, S. G. Kalashnikov, V. V. Kukushkin, and S. M. Ovechkin, Sov. Tech. Phys. Lett. 6, 237-238 (1980).

${ }^{31}$ D. M. Pozar, Microwave Engineering, 4th ed. (John Wiley \& Sons, 2012).

${ }^{32}$ Yu. A. Romanov, Opt. Spektrosk 33, 917 (1972).

${ }^{33}$ A. O. Selskii, A. A. Koronovskii, A. E. Hramov, O. I. Moskalenko, K. N. Alekseev, M. T. Greenaway, F. Wang, T. M. Fromhold, A. V. Shorokhov, N. N. Khvastunov, and A. G. Balanov, Phys. Rev. B 84, 235311 (2011).

${ }^{34}$ D. Hardwick, Ph.D. thesis, University of Nottingham, 2007.

${ }^{35}$ M. T. Greenaway, A. G. Balanov, E. Schöll, and T. M. Fromhold, Phys. Rev. B 80, 205318 (2009).

${ }^{36}$ A. E. Hramov, V. V. Makarov, A. A. Koronovskii, S. A. Kurkin, M. B. Gaifullin, N. V. Alexeeva, K. N. Alekseev, M. T. Greenaway, T. M. Fromhold, A. Patanè, F. V. Kusmartsev, V. A. Maksimenko, O. I. Moskalenko, and A. G. Balanov, Phys. Rev. Lett. 112, 116603 (2014).

${ }^{37}$ N. Alexeeva, O. Makarovsky, A. Patanè, M. B. Gaifullin, F. Kusmartsev, and T. M. Fromhold, Phys. Rev. Lett. 109, 024102 (2012).

${ }^{38}$ T. M. Fromhold, A. Patanè, S. Bujkiewicz, P. B. Wilkinson, D. Fowler, D. Sherwood, S. P. Stapleton, A. A. Krokhin, L. Eaves, M. Henini, N. S. Sankeshwar, and F. W. Sheard, Nature 428, 726-730 (2004).

${ }^{39}$ H. Willenberg, G. H. Döhler, and J. Faist, Phys. Rev. B 67, 085315 (2003).

${ }^{40}$ A. Balanov, N. Janson, D. Postnov, and O. Sosnovtseva, Synchronization: From Simple to Complex (Springer, 2009). 